David Murray

\title{
Ein ,volles Lied'. Übertragung und Klang am Beispiel der geistlichen Lieder des Mönchs von Salzburg
}

\section{Einleitung}

Neunundvierzig geistliche Lieder werden dem Mönch von Salzburg zugesprochen. ${ }^{1}$ Die Lieder, die am Hof des Salzburger Erzbischofs Pilgrim II. von Puchheim (reg. 1365-1396) entstanden, stellen eines der wichtigsten Korpora religiöser Lieder des deutschen Spätmittelalters dar. ${ }^{2}$ Sie bestehen zu fast gleichen Teilen aus Übersetzungen lateinischer liturgischer Hymnen und Sequenzen auf der einen Seite sowie Eigendichtungen des Mönchs ohne direkte lateinische Vorlage auf der anderen. Fast alle Lieder sind mit musikalischer Notation überliefert. Wie bei deutschsprachigen geistlichen Liedern allgemein häufig der Fall, scheint ihr künstlerischer Anspruch in der Forschung von anderen Aspekten gewissermaßen überschattet $\mathrm{zu}$ werden. Die Frage nach der möglichen liturgischen Nutzung solcher Lieder hat zum Beispiel von der ästhetischen Untersuchung des Repertoires abgelenkt. ${ }^{3}$ Die Stellung der geistlichen Lieder aus dem Mönch von Salzburg-Korpus in der Forschung wird auch durch das weitgehende musikwissenschaftliche Desinteresse an der spätmittelalterlichen Monophonie geprägt. ${ }^{4}$ Die Aufmerksamkeit der Musikwissenschaft hat eher den weltlichen Liedern des

1 (Dieser Beitrag entstand im Kontext des ERC-Projekts ,Music and Late Medieval European Court Cultures‘ an der Universität Oxford. Das Projekt wird von dem ERC im Rahmen des Forschungsund Innovationsprogrammes ,Horizont 2020‘ gefördert (Fördervertrag Nr. 669190). Neben den HerausgeberInnen möchte ich Henrike Lähnemann, Olivia Kobiela und Mai-Britt Wiechmann für ihre freundliche Anregung und sprachlichen Hinweise danken.)

Vgl. grundlegend Wachinger: Der Mönch von Salzburg. Wachinger hat mehrere der Lieder aus dem zentralen Mönch von Salzburg-Korpus ausgeschieden; vgl. Wachinger: Der Mönch von Salzburg. Zur Überlieferung, S. 119-137. Zur Identitätsfrage, vgl. Die weltlichen Lieder, S. 1-8. 2 Zu Pilgrim von Puchheim, vgl. Klein; zum erzbischöflichen Hof Salzburgs vgl. Schneider, bes. S. 71-83.

3 Zum eventuellen liturgischen Gebrauch der Salzburger Lieder, vgl. zuletzt Kraß, S. 218. Obwohl Johannes Janota 1968 verweigerte, volkssprachlichen geistlichen Liedern die Möglichkeit liturgischen Gebrauchs strictu sensu einzuräumen, haben jüngere Forscher die Lieder als allgemein „gottesdienstlich“ charakterisiert. Vgl. Janota und Rosmer: Der Mönch von Salzburg und das lateinische Lied.

4 Eine wichtige Ausnahme hier ist die Arbeit von Stefan Engels; vgl. Engels.

Ә OpenAccess. ( 2020 David Murray, publiziert von De Gruyter. (cc) BY Dieses Werk ist lizenziert unter der Creative Commons Attribution 4.0 International. https://doi.org/10.1515/9783110666816-006 
Korpus gegolten, soweit diese zu den frühesten überlieferten Belegen der nichtliturgischen Mehrstimmigkeit im deutschsprachigen Raum zählen. ${ }^{5}$ Dementsprechend ist die Musik der ausschließlich einstimmigen geistlichen Lieder seitens der Forschung nur selten gleichberechtigt behandelt worden, obwohl sie die Entstehung, Überlieferung und das ,Leben' der Lieder weitgehend mitbestimmt.

Dieser Beitrag zielt deswegen darauf ab, das Klangliche enger einzubeziehen, indem er die geistlichen Lieder des Mönch von Salzburg-Korpus als musikalischtextliches Gesamtkunstwerk untersucht. Damit möchte ich über die Dichotomie von Sprache und Musik hinausgehen, die auf die verankerte Auffassung zurückgeht, dass Musik vor der Renaissance weder als künstlerischer Ausdruck noch als semantisch befrachtet betrachtet werden könne. ${ }^{6}$ Nur der Text eines Liedes habe einen feststellbaren Sinn, während seine Melodie letzten Endes ,blind' sei. ${ }^{7}$ Die vermeintliche Kluft zwischen Sprache und Musik verhindert also den ganzheitlichen Blick auf das geistliche Lied im Einzelnen und das geistliche Singen im Allgemeinen. ${ }^{8}$ Im Mittelalter schloss die musica mundana fast selbstverständlich die menschliche Stimme ein. Die untrennbare Verbindung aus Stimme, Wörtern und Melodie wird beispielsweise von Eustache Deschamps belegt, indem er die Fähigkeit eines prince du puy, den Text von der Melodie abzulösen, um ein Lied niederzuschreiben, als besonders bemerkenswert qualifiziert. ${ }^{9}$

Neuere Entwicklungen in der Musik- und Literaturwissenschaft lassen jedoch vermuten, dass ein einheitlicherer Ansatz zum mittelelterlichen Lied möglich ist. Auf der einen Seite hat Wulf Arlts Plädoyer, sowohl der Melodie wie auch dem Text semantischen Wert beizumessen, Anlass für eine Handvoll Versuche in diese Richtung gegeben. ${ }^{10}$ Andererseits hat Markus Stock anhand der Lieder Gottfrieds von Neifen einen radikalen Wandel in der Poetik des dreizehnten Jahrhunderts nachgewiesen. ${ }^{11}$ Stock zufolge benutzt Gottfried Schlüsselwörter, ohne sich ausschließlich auf deren Semantik zu beziehen. Klang und Inhalt bilden demzufolge

\footnotetext{
5 Vgl. etwa Welker.

6 Den angeblich minderen Sinngehalt der vormodernen Musik betont bspw. Ludwig Finscher; vgl. Finscher, S. 15.

7 Zur ,Blindheit‘ der Musik im Allgemeinen, vgl. Zumthor, S. 145.

8 Hier sei daran erinnert, wie die eigene Sprache die Auffassung eines Phänomens prägt. Wo das Deutsche eine klare Trennung zwischen dem einzelnen „Lied“ und der breiteren Kategorie des „Gesangs“ als die Produkte des Verbums „singen“ macht, sind diese auf Englisch mit demselben Wort „song“ bezeichnet; sie sind dementsprechend vielleicht einfacher als Teile eines Großphänomens aufzufassen.
}

9 Haug, S. $62 \mathrm{f}$.

10 Vgl. Arlt und, später, Schadendorf und Kandler.

11 Vgl Stock. 
eine gemeinschaftliche Botschaft. ${ }^{12}$ Auch in der Mönch-Forschung hat Dagmar Hirschbergs Aufsatz zu Dy trumpet (W5) überzeugend dargelegt, welch fundamentale Bedeutung Melodie, Klang und musikalische Gestaltung für die Komposition eines Lieds als klingende Einheit haben. ${ }^{13}$

Der Terminus ,Klang‘ hat in der neueren germanistischen Forschung und vor allem in Untersuchungen zum deutschen Minnesang eine gewisse Konjunktur. Er ist besonders wichtig im Kontext des geistlichen Liedes, weil er eine Brücke zwischen den üblicherweise separat konzipierten Elementen der Liedkunst schlägt. Klangproduktion und -rezeption erfolgen sowohl auf textliche wie auch auf melodisch-musikalische Weise. Diese neuere Forschungstendenz betont den wichtigen aber flüchtigen Beitrag des Klanglichen zum Sinngehalt der mittelalterlichen Lied- bzw. Dichtkunst. ${ }^{14}$ Susanne Köbele fasst dies so zusammen: „Klangkunstwerke sind auf eine analytisch nur schwer fassbare Weise von Semantik entlastet, ohne athematisch, asemantisch oder gar Unsinn zu sein“. ${ }^{15}$ Köbeles Aufsatz spiegelt in dem Sinne die zeitgleiche Blüte des breiteren interdisziplinären Diskurses der Sound Studies wider. ${ }^{16}$ Die gängige Auffassung des Klangs in dieser germanistischen Forschungsströmung bleibt jedoch weitgehend sprachlich orientiert. ${ }^{17}$ Bei einem grundsätzlich textbezogenen Ansatz bleiben Faktoren wie Klang und Musik und ihr Anteil am Erleben und Erinnern eines Liedes weitgehend unberücksichtigt. Erlebnis ist von entscheidender Wichtigkeit im Bezug auf das gesungene Lied. ${ }^{18}$ Singen impliziert nämlich viel mehr als andere Kunstformen einen tief ruhenden Einklang mit dem Körper, der sich der üblichen wissenschaftlichen Analyse entzieht. ${ }^{19}$ Vor diesem Hintergrund können Wert und Sinn eines Liedes im Einzelnen und der gesamten Singkultur einer Gemeinschaft im Allgemeinen nicht ohne Weiteres auf eine objektive Größe reduziert werden. ${ }^{20}$ Die Irreduzibilität der Bedeutung eines Liedes lässt sich auch für die geistliche Kultur des Mittelalters im Ganzen vorbringen, und sie wird in

12 Stock, bes. S. 199f.

13 Vgl. Hirschberg.

14 Vgl. Köbele, Braun und Schneider: Poetik des Klangs; dies.: Er liez ze himel tougen erhellen sîner stimme dôn.

15 Köbele, S. 319.

16 In der Mediävistik außerhalb der Germanistik, vgl. Dillon, Williamson.

17 Eine wichtige Ausnahme bildet die Studie von Caroline Emmelius.

18 Im Folgenden gehe ich davon aus, dass alle besprochene Lieder fürs Singen konzipiert waren. 19 Vgl. Gibbs, S. 2: „People's subjective, felt experiences of their bodies in action provides part of the fundamental grounding for language and thought. [...] We must not assume cognition to be purely internal, symbolic, computational, and disembodied, but seek out the gross and detailed ways that language and thought are inextricably shaped by embodied action“.

20 Zumthor, S. 133. 
manchen Zusammenhängen zunehmend anerkannt. ${ }^{21}$ Andreas Haug betont z. B. die „Abstimmung der melodischen Parameter des Lieds mit dem in ihm besungenen Gegenstand, die sein ästhetisches Format bestimmt“. ${ }^{22}$ Gerade diese wie auch andere noch subjektivere Abstimmungen untermauern die Kohärenz und Einheitlichkeit eines Liedes. Das sinnstiftende und sinntragende Potenzial des Klanglichen darf in diesem Zusammenhang nicht übersehen werden. ${ }^{23}$

Vor diesem Hintergrund bieten die verschiedenen Übersetzungen, die in den geistlichen Liedern des Mönches von Salzburg hervortreten, ein vielversprechendes Feld, um die unterschiedlichen Achsen der mittelalterlichen Liedkultur zu untersuchen, die Wert und Sinn transportieren können. Die Lieder sind bislang nur auf den textlichen Sinngehalt gelesen worden. Um die Vielfalt klanglicher Sinnstiftung in den Übersetzungen des Mönchs-Korpus vorzuführen, werden im Folgenden drei Beispiele diskutiert. Sie weisen jeweils eine besondere Beziehung zwischen dem Mönchs-Lied und seiner Vorlage auf. Im ersten Fall lässt sich an der Übersetzung des Ave virginalis forma die untrennbare Beziehung zwischen stimmlichen Klängen, Text und Melodie ablesen und wie diese die spätere Überlieferung prägt. In der zweiten und dritten Gruppe wende ich mich einer anderen Art von klingender bzw. musikalischer Übertragung zu, nämlich der Kontrafaktur der Sequenz Mundi renovatio und den vermutlichen Kontrafakturen auf die Lieder Seht an die heide Gottfrieds von Neifen und Mich jâmert ûz der mâze Wernhers von Hohenberg. Insgesamt veranschaulichen diese Stücke, wie die Melodie und die akustischen Elemente eines Liedes zu einem einheitlicheren Verständnis des Lebens und möglicherweise auch der Wirkung des volkssprachlichen geistlichen Lieds führen können.

\section{Die swär in dewtsch-Lieder}

Mein erstes Beispiel könnte man in Anlehnung an die Rubrik, die sich in der vermutlich aus dem Kloster Tegernsee stammenden Handschrift A (München, BSB, Cgm. 715) findet, als ein swär in dewtsch-Lied bezeichnen. Mit diesem Titel versieht der Rubrikator des Überlieferungsträgers vier deutsche Lieder (G 7, G 26, G 39 und G 47), die in Formulierung, Konstruktion und anderen Elementen der la-

21 Den Erlebnisraum besser in die mediävistische Forschung einzubinden war eins der Hauptziele des Projekts ,Experience of Worship in late medieval Cathedral and Parish Church' an der Universität Bangor; vgl. Harper und www.experienceofworship.org.uk (25. April 2019).

22 Haug, S. 101.

23 Neben Arlt, vgl. Fassler, bes. Kap. 12 und 13, zum Bedeutungspotenzial des Viktorinischen Sequenzenrepertoires. 
teinischen Vorlage äußerst genau folgen. ${ }^{24}$ Die Kunstfertigkeit dieser textgetreuen Übersetzungen ist bereits von Gunther Bärnthaler und Ingo Reiffenstein kommentiert worden. ${ }^{25}$ Bärnthaler hat die Lieder, die ihre lateinische Vorlage formal und stilistisch genauer wiedergeben, von anderen abgegrenzt, die formal freier aber inhaltlich präziser sind. Die letztgenannte Gruppe sollte den Text also einem lateinunkundigen Publikum eröffnen. Die swär in dewtsch-Liedtexte beweisen hingegen sowohl die Hochschätzung von sprachlicher und formaler Virtuosität als auch das Bemühen des Salzburger Dichters, das lateinische Lied in seiner komplexen Gesamtheit zu übermitteln. Paradebeispiel für diesen übersetzungstechnischen Ansatz ist das Ave bis grüßt, megdlich forme (G5), eine Verdeutschung von Jakobs von Mühldorf Sequenz Ave virginalis forma (Anal. hymn. 54, Nr. 243, S. $379-382) .{ }^{26}$ Die Übertragung weist systematische und abgestufte Übernahmen aus dem Lateinischen auf. Ich zitiere den Text der stilistisch äußerst anspruchsvollen lateinischen Sequenz, den die Handschrift A als ein swäres Latein bezeichnet, nach der Handschrift München, BSB, Cgm. 716, fol. 90 ${ }^{\mathrm{v}}-94^{\mathrm{r}}$. Daneben gebe ich Burghart Wachingers Ausgabe der Übertragung des Mönchs nach der Handschrift U (Berlin, SBB, mgo 137, fol. 158 $-160^{\mathrm{v}}$ ) wieder. $^{27}$

\author{
Ave, virginalis forma, \\ Deitate plena norma, \\ Agni Syon templum. \\ Botrus cipri balsamatus, \\ Austro pneumatis perflatus \\ In pacis exemplum. \\ Chere numen in prophetis, \\ Vere lumen, pax in metis, \\ Regnans virtus alma. \\ Dei caritate culta, \\ Spei claritate fulta, \\ Pregnans mirtus palma.
}
Ia Ave bis grüßt, megdlich forme, der gotheit ervolte norme, lammes Sion tempel.
Ib Bintrub cyper balsam-tüftet, die gots geist süß hat erlüftet in frides exempel.

Clar grüßt vol geist in propheten, gar süßt vol- leist, frid in steten, blünder iugent palme.

IIb Der göt- li- chen schön ein zirde, ger nöt- li- chen frön sin wirde, gründer tugent galme.

24 Die G-Nummern beziehen sich im Folgenden auf die Ordnung der geistlichen Lieder des Mönchs in Spechtlers Ausgabe; vgl. Die geistlichen Lieder.

25 Vgl. Bärnthaler und Reifenstein. Vgl. auch März zur Ut queant laxis-Übersetzung, Das hell aufklimmen deiner diener stimmen (G 47).

$26 \mathrm{Zu}$ Jakob von Mühldorf, vgl. Die geistlichen Lieder, S. $17 \mathrm{f}$.

27 Wachinger: Der Mönch von Salzburg. Zur Überlieferung, S. 140 -144; die Verseinteilung des lateinischen Textes ist hier an den deutschen Text angepasst. 
Engadina vitis mitis,

Precium in margaritis,

Casta generacio.

Forma que senile yle

Esse duxit ad subtile

Noys veneracio.

Gabaon in victoria,

Sol lucens in gloria,

Iosue victoris.

Honor throni gracie,

Eous in facie,

Cornuti lictoris.

Iaspidina preclaritas

Quam fides polivit,

Aspidina disparitas

Quam numquam attrivit,

In cor Eve sata.

Karissima deliciis

Affluens natura,

Clarissi[m]e viciis

Est palme statura

Tua similata

Leonis vox mox suscitans

Nos ab Ade clade

Fenicis fos nos renovans

A letali mali

Primevo prohemio

Maceria qua latuit

Pellicanus sanus

Unicornis vis patuit

Mortis virgo pirgo

Tuo casto gremio.

Noemi sine mara,

Clara cara gnara Sara, paradysus

In qua deus homo visus

Expers omnis criminis.

Orthodoxa fermata,

Nata grata data rata sata soli

Regens cardines ac poli, Stupor Dagon liminis.
IIIa Engadi beschnitten sitten winreb, lust in margaritten, küscher küsche küsch geburd.

IIIb Forme, die materge erge braht in höhster wesen kerge. götliche wird, e ihtsiht wurd.

IVa Gabaon der stat sigeswunne, sighaftiger sigessunne Josue des kempfen.

IVb Höhste wird der gnaden throne, sonn in mittel brehndem frone Moisi on tempfen.

Va Jaspis du stein, den der gloub hat reinlich gepoliret.

des slanges schein mit brödem rat

den hat nie vergiret,

der in Eve herz gespan.

Vb Karissima, liebst aller lieb, wünschelfluß naturen, die reinste, ja on sünden stieb, din palm den figuren wol sich zugelichen kan.

VIa Lebendig le- schre, der uns tunget von Adame schame.

des fenix glut frut uns junget

von tödlichem krichen,

das von erst her uf uns doß.

VIb Megdlich steinwant, stant nam in dir pelicane sane,

einhurnes sin in fluchtes gir

todes freide, meide

zart, in diner küschen schoß.

VIIa Noemi du bist, die schön, on bitter gar; zwar war Sar klar; par- adise, in dem got mensch kom zu wise fri vor aller sünden spür.

VIIb Ordens rechte firmunge der trinitat, rat stat, pfat, sat. nat- ürlichen erd und himelechs dir wichen, schrickt uf Dagon under tür. 
Porta clausa quam transivit

De Bosra formosus,

Cuius pausa te sancivit,

Ut flos fructuosus esses

Fragrans omnia.

Quis appendit terre molem

Digitis tris dei?

Qui comprendit patris prolem?

Dic o mater spei:

,Sancta ego domina'.

Rubus moyisi fiscella, Incombustus in procella, Mundi nans immobilis.

Splendor patris et figura, Factor factus creatura, Tua virgo nobilis.

$\mathrm{Tu}$, caritate media, Thronus es constratus, Tu cosmi tollens tedia, Noe demonstratus, Pace tipus Iridis. Virgo Maria domini Sabbaoth electa, Tu unica spes homini Mentumque delecta, Iacob strophe viridis.

Xpisti Iesu mater, ave, Mortis anxioma grave Dum advenerit, da suave Vesper, reos ad conclave Collocans yerarchicum. Yesse virga veni veni, Duc in Bethel ductu leni Ubi psallunt quarter seni. Sense nova laude pleni Euphonos seraphicum.
VIIIa Port vercluset, die durchsleiche von Bosra der schöne. rein dich puset sin umbreiche, daz ein blum in fröne du werst brehnder selikeit.

VIIIb Quis wer henget an erd laste gots drien vingern einer? iht wer fenget vaters glaste? o sprich, muter reiner hoffnung: ,ich frau, frau und meit‘.

IXa Rötend Moisi stud, zistel, unverprant, in der werlde mistelflut die schwam on sünden rür.

$\mathrm{IXb}$ Schin des vaters und figure, schöpfer wart din creature, edle magt megdlicher kür.

Xa Tu rechter lieb mit reiner mitt bist ein thron bestrete. $\mathrm{du}$ hast der werlde treg verquitt, Noe zeichen stete, fridlich regenbog, erkant.

$\mathrm{Xb}$ Vrau magt Mari, des herren Sabaoth erkesen.

du winreb on alles werren, menschen hoff, süß wesen. Jacob list din kind ervant.

XIa Xpisti Jhesu muter grüßig, wenn des todes not unmüßig mit uns werd, so tu schwer büßig. sur we mach uns, muter, süßig in den thrönen seraphi.

XIb Yesse fron gert, kum, kum schiere. für hin z' Bethel uns zu dire, do da singent sehsstund viere altherren. eia, da ziere uns in chören ierarchi. 
Zelotem deum patrem ora,

Nato iube sine mora,

Amborum flamen implora,

Ut uniti sint in prora,

Dum transimus rubrum mare.

Illic, iacob stella, clare,

Ut possimus transfretare

Tecum leti post examen.

Fiat, fiat, Amen, Amen.
XII Zart guldin, got vater flege,

schaff mit got sun durch vermege,

got ir beider geist erwege,

daß er ein des schiffes pflege

durch des wilden meres trone,

do lücht Jacobs sterne frone,

daß wir himelschiffen schone

vor gericht in dinem namen.

das gescheh, das! Amen. Amen.

Neben der unvermeidlichen Übernahme von Eigennamen wird hier eine beträchtliche Anzahl von Substantiven direkt übernommen (vgl. z.B. in Versikel I: forme, norme, tempel und exempel). Ebenfalls werden an anderen Stellen, auch wo es keinen Anlass in der Vorlage gibt, latinisierende Lexeme in den deutschen Text aufgenommen (z. B. materge IIIb, figuren $\mathrm{Vb}$ ).

Weiterhin bemüht sich der Autor, die Phoneme seiner lateinischen Vorlage akribisch wiederzugeben. In seiner wegweisenden Studie, Vom Kult zur Kunst' hat Bruno Quast diese Bewahrung von Vokalen aus der lateinischen Vorlage als „Positionalität“ bezeichnet. ${ }^{28}$ Dieser poetische Ansatz sei ein Versuch, den Körper des rituellen bzw. liturgischen Textes unangetastet $\mathrm{zu}$ bewahren und weiterzutradieren. Quast betont damit den unschätzbaren Stellenwert der Ganzheitlichkeit eines Liedes im Rahmen der mittelalterlichen religiösen Kultur. So wird das abecedarische Raster von Ave virginalis forma sorgfältigst übernommen, auch wenn das zur direkten Übernahme von Wörtern aus dem Lateinischen (Karissima, Vb) führt. In einem seltsamen Fall wird das lateinische Wort mit einem deutschen verdoppelt (Quis wer hengt an erd last, VIIIb). Diese Akribie als rein textbezogen zu betrachten, missachtet aber die eigentlichen Charakteristika eines Liedes, denn bei einem Lied handelt es sich nicht nur um ein rein textliches Objekt, sondern um die gesungene Verwirklichung des Textes in einer bestimmten Aufführungssituation. Diese kontextbedingte performative Verwirklichung einschließlich der Gesten des Vortragenden nennt Paul Zumthor das œuvre. ${ }^{29}$ Wesentlicher Bestandteil des œeuvres ist neben einem Rezipienten also auch die Melodie. Im Fall des Ave virginalis forma trägt die ausgeschmückte Melodie erheblich zum gesamtheitlichen Erlebnis des Liedes bei. Die Interaktion von sprachlichem und musikalischem Schmuck führt zu einem mehrdimensionalen Bedeutungspotenzial, das sich gut am Beispiel des siebten Doppelversikels des Ave grüest pist veranschaulichen lässt. Hier werden mit besonderer Feinheit die Vokale des raf-

28 Quast, S. 154-166, hier S. 164. Quast bespricht auch die Salzburger Übersetzung des Hymnus Ut queant laxis (G 47), S. 146-154.

29 Zumthor, S. 92-94. 
finierten lateinischen Vorlagentextes in die Volkssprache überführt. ${ }^{30}$ Diese sind im folgenden Abdruck fett gesetzt:

Noemi sine mara

Clara cara gnara sara Paradysus In qua deus homo visus Expers omnis criminis Orthodoxa fermata Nata grata data rata sata soli Regens cardines ac poli Stupor dagon liminis
Noemi du bist, die schön, on bitter gar;

zwar war Sar klar; par- adise, in dem got mensch kom zu wise fri vor aller sünden spür. Ordens rechte firmunge der trinitat, rat stat, pfat, sat, nat- ürlichen erd und himelechs dir wichen, schrickt uf Dagon under tür.

Mit Blick auf die Melodie wird aber deutlich, dass der Text nur die halbe Sache ist, denn zum einen ergänzt die melodische Form die textlichen Verzierungen und zum anderen unterstreicht sie die Simplizität der absichtlich monoton gehaltenen Textformulierungen. Ich gebe die Melodie nach der Handschrift E (Wien, ÖNB, Cod. 4696, ff. 159r-167r) wieder:
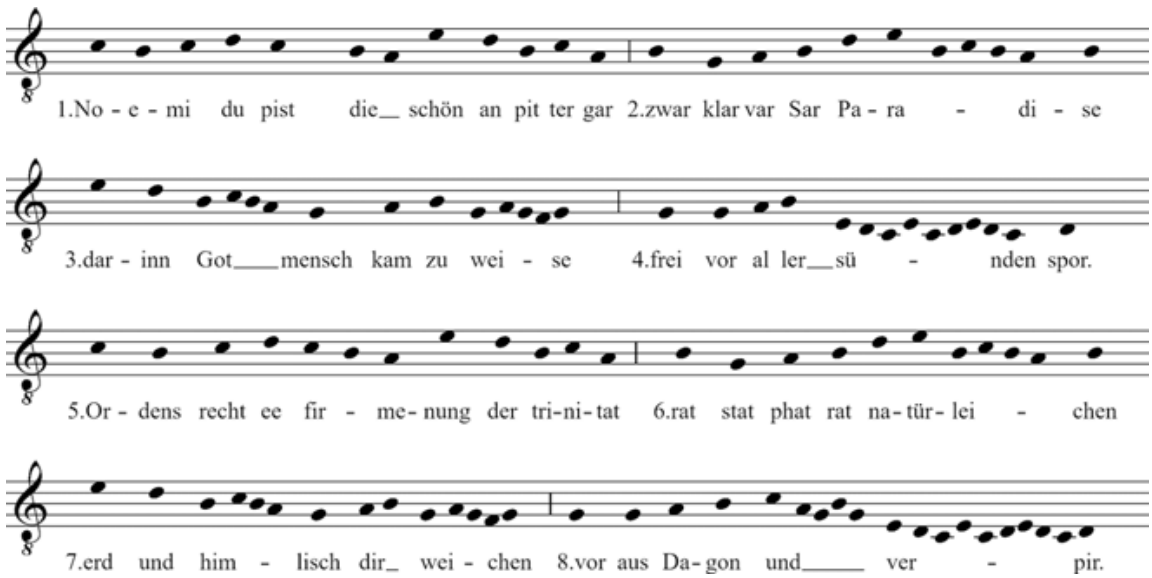

Notenbeisp. 1: Ave, grüest pist, magtleich forme, Versikel VII nach Hs. E

Anmerkung: Hs. E lässt im vierten Vers des VII. Versikels die Notenreihe $c-a-g-b-a$ aus (vgl. Vers 8 unten zu ,und'). Hs. A, die melodisch weniger zuverlässig ist, lässt dieses Melisma auch beiseite, aber versetzt die Notenreihe e-d-c-e auf der ersten Silbe von ,sünden“ um eine Quinte nach oben.

30 Zur Poetik des Ave virginalis forma, vgl. Szövérffy, S. 62. Er beschreibt diese Sequenz als ein Beispiel der „mehr oder weniger manieristischen Leichdichtung“. 
Augen- bzw. ohrenfällig in diesem Doppelversikel ist also die ungebrochene Verbindung der längeren Melismen am Ende des vierten und achten Verses, zu denen Paradysus und visus erklingen, mit einem einzigen Vokal. Im zweiten Vers des Versikels hingegen ist die Textur der Melodie syllabisch, und eine ganze Reihe von Vokalen wird genau wiedergegeben (zwar war Sar klar; par-adise und rat stat, pfat, sat, nat- ürlichen, VIIa,2 bzw. VIIb,2). Als Ganzes betrachtet stellt Ave grüest pist ein schönes Beispiel für die kompositorischen Möglichkeiten dar, die dem Lieddichter zur Verfügung standen. Die enge und grundlegende Beziehung zwischen der musikalischen Faktur eines Liedes und der Übertragungsarbeit kann also nicht genug betont werden.

Diese swär in dewtsch-Stücke stellen also nicht nur Übertragungen eines Textes oder seines Inhalts dar, sondern auch die Übertragung jenes klingenden Erlebnisses bzw. jener körperlichen Handlung, die diesem Erlebnis zugrunde liegen. Sie reagieren damit auf die Grundprinzipien der Vorlage, denn sowohl das Ave virginalis forma im Einzelnen wie auch die Sequenzenform im Ganzen, die sich aus der Verlängerung und Verzierung des letzten As des Alleluia entwickelte, beruht auf der Tragkraft klingender Vokale. Sie bewahren also nicht nur das rituelle, textliche Objekt, sondern auch dessen tiefere handlungsbezogene Bedeutsamkeit. Wie schon angedeutet, kann dieser Wert nur im Rahmen der Performanz und damit physisch entfaltet werden. Diese Körperlichkeit liegt dem gemeinschaftsstiftenden Sinn des rituellen Singens zugrunde, sei es im Kontext des physisch kommunalen Singens oder in dem Bewusstsein, das ein Lied von anderen Menschen, die zwar körperlich nicht anwesend, aber spirituell verwandt sind, gesungen wird. ${ }^{31}$ In diesem Sinne sind swär in dewtsch-Lieder, die ein nicht klerikales Publikum fernhalten zu wollen scheinen, in der Tat radikaler ,demokratisierend' als rein text- bzw. inhaltsorientierte Übersetzungen. Denn der Versuch in den swär in dewtsch-Liedern, geistliche Lieder als klingende Einheiten weiterzutradieren, eröffnet einem lateinunkundigen Publikum ein Erlebnis, das dem ursprünglichen Lied viel nähersteht als in den Liedern, die allein den Inhalt übermitteln.

\section{Mundi renovatio und die mittelalterliche Kontrafaktur}

Man muss aber auch über die Ganzheitlichkeit des einzelnen Lieds hinausblicken. Eine bestimmte Melodie hat in der Erfahrung des Rezipienten eine kaum ablös-

31 Zur Rolle des klingenden Körpers in der geistlichen Gemeinschaft, vgl. Jones, S. 235 f. 
bare Beziehung zu einem bestimmten Liedtext. Die mittelalterliche Liedkunst war aber kein statisches Feld, und Dichter scheuten sich nicht, metrische Raster und Melodien aufzunehmen. Die Ganzheitlichkeit eines einzelnen Liedes war also langfristig nicht unantastbar. Wie der Fall von Ave grüest pist veranschaulicht hat, ist es unmöglich, die einst in sich geschlossene Liedeinheit im Nachhinein in Musik und Text zu teilen, ohne dass dies zwangsläufige Spuren im jeweils anderen Bestandteil hinterließe. Vor diesem theoretischen und praktischen Hintergrund muss man daher auch die beliebte kompositorische Praxis der Kontrafaktur betrachten. In der Kontrafaktur werden neue Texte komponiert, die einer schon bestehenden Melodie zugeordnet werden. Damit wird dem Dichter bzw. Komponisten ein breites Feld für inhaltliche Bezugnahmen und Anspielungen eröffnet. ${ }^{32}$ Von der Rezipientenseite aus betrachtet, überträgt die Melodie also bestimmte Resonanzen einer vormals geschlossenen Liedeinheit auf einen neuen Zusammenhang. Welche Resonanzen dabei genau auf das neue Lied übertragen werden, hängt in hohem Maße vom individuellen Zuhörer, seiner Erfahrung und seinen Kenntnissen $a b .{ }^{33}$ In diesem Sinn entziehen sich die Beweggründe für die Kontrafaktur oft den Normen wissenschaftlicher Analyse. Gleiches gilt auch für die Beurteilung, ob ein Versuch Neues zu Altem zu setzen erfolgreich war, da dies ebenfalls nur vom einzelnen Rezipienten beurteilt werden kann.

Unter diesem Blickwinkel nahm das Lied Sälig sei der selden zit (G 17) in der Forschung eine problematische Stellung ein. Das Lied ist in drei von acht Handschriften unter der Melodie des Mundi renovatio (Anal. hymn. 54, Nr. 148, S. $224-$ 227) überliefert. Es knüpft jedoch textlich nicht direkt an die Sequenz an. Hans Waechter und Franz Viktor Spechtler nennen das Stück in ihrer Musikausgabe daher „eine freie Dichtung auf die Ostersequenz“. ${ }^{34}$ Die Ostersequenz, die wahrscheinlich im Pariser Viktoriner-Kloster entstand, war in Salzburg und dem südostdeutschen Raum vor allem in einer fragmentierten Version bekannt. ${ }^{35}$ Sie beschreibt die Auferstehung Christi anhand von Frühlingsmetaphorik (im zweiten Versikel heißt es etwa Resurgenti Domino, Conresurgent omnia). Im Gegensatz zum Mundi renovatio ist das Sälig sei der selden zit ein Lobpreis auf die Gottesmutter als

32 Vgl. Gruber, der Interaktionen im Rahmen der Kontrafaktur zwischen Dichtern romanischer Sprachen als grundsätzlich wettbewerbsorientiert auffasste.

33 Zur Frage mehrerer überlappenden Rezipientenkreise eines Liedes, vgl. Murray.

34 Waechter, S. 250.

35 Vgl. AH 54, S. 226. Sie war auch Objekt einer wahren Übersetzung im Mönch von SalzburgKorpus ( $\mathrm{G}$ 28, Aller werlde gelegenhait). Zu G 28 wie auch der Übersetzung Oswalds von Wolkenstein, vgl. Straub. 
Vermittlerin der Ostergeschehnisse. Ich gebe den Text nach Spechtlers Ausgabe wieder. $^{36}$

I Sälig sei der selden zeit, an der all mein frewde leit, wann der liebe Jesus Christ von dem tod erstanden ist. alle dingk vernewet sind, juden gelaub der ist nu plint; sei gelobt, der magde kind.

II Christen und die kristenhait haben in got sicherhait; wer hie klagt sein missetat und in frewden lebt noch rat, der hat dort vor aller not. frid uns vor der helle tot und auch speis mit der engel brot.

III Himmel tuer in offen ist, sehent sie an underfrist Jesum und die mueter sein, die trait himmelischen schein, wenn in ir verslossen lag aller engel ostertag; niemant sie volloben mag.

IV Sie ist aller engel lob, sie ist der steren von Jacob, grüener pusch, der nie verpran. Salomon der weise man geleichet sie zu seinem thron und zu der gerten hern Aaron, und zwelf stern leuchten in irer kron.

V Daniel sach einen pergk, einen stain an mannes werk, Gedeon zaigt uns sein fel und sein porten Ezechiel, David mit der herphen sein lobt mit mir die frawen mein, lob sei ir von mir gesait, gelobt sei all ir wirdikait.

36 Edition: Spechtler, Die geistlichen Lieder, S. 206-208. 
Der Text weist hier eine gewisse Leerstelle auf, insofern Maria erst nach den ersten beiden Doppelversikeln, die zum einen die Auferstehung und den Frühling betonen und zum anderen das Heil für alle Gläubige behandeln, in Erscheinung tritt. Der Betende muss auf den dritten Versikel warten, bis Jesu[s] und die mueter sein endlich auftreten. Erst dann widmen sich der vierte und fünfte Versikel der Mutter Gottes. Wie vielfältig die sich daraus ergebenden Zuschreibungsmöglichkeiten waren, spiegelt sich in der handschriftlichen Überlieferung des Lieds wider, insbesondere in den Rubriken, die dem Sälig sei der selden zeit vorausgehen.

\begin{tabular}{|c|c|c|c|}
\hline Hs. & Siglum & Rubrik & Notation \\
\hline A & $\begin{array}{l}\text { München, BSB, } \\
\text { Cgm. 715, fol. } 141^{r}\end{array}$ & Zw Ostern & $\mathrm{Ja}$ \\
\hline B & $\begin{array}{l}\text { München, BSB, } \\
\text { Cgm. 1115, fol. } 30^{r}\end{array}$ & $\begin{array}{l}\text { Von vnnser lieben frawe(n) die Sequenczn zw } \\
\text { Österleicher czeit }\end{array}$ & $\mathrm{Ja}$ \\
\hline $\begin{array}{l}\text { C } \\
(\operatorname{Reg})\end{array}$ & $\begin{array}{l}\text { München, BSB, } \\
\text { Cgm. } 628, \text { fol. } 1^{r}\end{array}$ & Von vnser fraue(n) zu der österlich(e)n czeit & - \\
\hline C & $\begin{array}{l}\text { München, BSB, } \\
\text { Cgm. } 628 \text {, fol. } 250^{\vee}\end{array}$ & Von vnser lieben frawn zw der osterlicher zeit & Ja \\
\hline D & $\begin{array}{l}\text { Wien, ÖNB, Cod. } 2856 \text {, } \\
\text { fol. } 236^{\text {r }}\end{array}$ & Von unser frawen & $\mathrm{Ja}$ \\
\hline $\mathbf{F}$ & $\begin{array}{l}\text { Wien, ÖNB, Cod. } 2975 \text {, } \\
\text { fol. } 155^{r}\end{array}$ & $\begin{array}{l}\text { Se(quitur) de b(ea)ta v(ir)gi(n)e p(salmus) pasca sub } \\
\text { melodia mundy r(e)nouacia }\end{array}$ & Nein \\
\hline G & $\begin{array}{l}\text { München, BSB, } \\
\text { Clm. } 4423 \text {, fol. } 55^{r}\end{array}$ & Von vns(er) lieb(e)n frawn zw ost(er)lich(er) zeyt & Nein \\
\hline J & $\begin{array}{l}\text { Wien, ÖNB, Cod. } 3741 \text {, } \\
\text { fol. } 16^{v}\end{array}$ & Von vnns(er) lieben frawn zü osterlich(er) zeit & Nein \\
\hline $\mathbf{E}$ & $\begin{array}{l}\text { München, BSB, } \\
\text { Cgm. 5249/59b, fol. } 2^{v}\end{array}$ & Mvndi Renouacō & Nein \\
\hline m & Graz, UB 271 , fol. $245^{\vee}$ & [keine Überschrift] & Nein \\
\hline $\mathbf{N}$ & $\begin{array}{l}\text { München, BSB, } \\
\text { Clm. 9656, S. } 116\end{array}$ & Mundi & Nein \\
\hline
\end{tabular}

Die unterschiedlichen Verknüpfungen, die die Rubriken bezeugen, lassen sich in vier Gruppen aufteilen. (1) Hs. A bezieht sich direkt auf Ostern und verzichtet damit auf weitere Spezifikationen. (2) Hss. BCGJ nennen Ostern zusammen mit Maria und entsprechen damit eher der Hervorhebung der Gottesmutter im volkssprachlichen Text. (3) Hs. D, die berühmte Mondsee-Wiener Liederhandschrift, geht mit dem Vermerk unser frawen einen Schritt weiter und verdrängt 
jeden Hinweis auf Ostern. Diese drei möglichen Auffassungen des Sälig sei der selden zeit spiegeln eine langsame Verschiebung des Fokus von der Auferstehung Christi auf seine Mutter hin wider. ${ }^{37}$ Etwas anders sind hingegen die Hss. E und N gelagert, die explizit auf die lateinische Formvorlage hinweisen. Auf die Frage, ob die beiden Vermerke als einfache Erinnerungen an die Melodie zu betrachten sind, komme ich gleich zurück. Die Rubrik in Hs. F bringt die Tendenzen zusammen, einerseits die Anwendung der Sequenz explizit zu erklären und andererseits nur auf die melodische Vorlage hinzuweisen und damit die österliche Verwendung des Lieds festzustellen. Die letztgenannten Hss. FEN sind somit ein sprechendes Beispiel für die virtuelle Mehrstimmigkeit, die durch die Kontrafaktur zustande kommt. ${ }^{38}$ Gleichzeitig ist die virtuelle Mehrstimmigkeit aber auch eine Voraussetzung der Kontrafaktur, denn wenn auch nur ein Teil des Publikums nicht fähig gewesen wäre, die ursprüngliche lateinische Sequenz beim Erklingen des Sälig sei der selden zeit auch inhaltlich ,mitzuhören“ und ,mitzudenken', dann wäre das kompositorische Verfahren zwecklos gewesen. ${ }^{39}$

Besonders augenfällig an der Ostersequenz Sälig sei der selden zeit ist, dass der Salzburger Dichter sie als Basis für eine Meditation auf die alttestamentarischen Vorversprechungen der Jungfrau Maria auswählt. Ostern ist nämlich in erster Linie die Feier der Erfüllung der göttlichen Prophezeiung. Dass die Gottesmutter, die nur am Rande der biblischen Ostererzählung steht, hier in den Vordergrund gerückt wird, ist ein typisches Beispiel für die langsame Verschiebung des Fokus, die prägend für die Entwicklung der mittelalterlichen geistlichen Kultur ist. ${ }^{40}$ So besteht die zweite Hälfte der deutschen Sequenz größtenteils aus einer Reihe marianischer Attribute: brennender Dornbusch (IV,3, vgl. Ex. 3,2-4); Thron Salomos (IV,5, vgl. Cant. 3,7) oder stain ane mannes werk (V,1-2, vgl. Dan. 2,34). Wie Spechtler in seiner Ausgabe zeigt, sind diese Sinnbilder feste Bestandteile eines beliebten und oft benutzten Motivrepertoires des Salzburger Dichters. ${ }^{41}$ Der Grad der Personalisierung steigt erst in den letzten vier Versen des Liedes an:

David mit der herphen sein

lobt mit mir die frawen mein,

$37 \mathrm{Zu}$,Aufmerksamkeitsverschiebungen“ in der mittelalterlichen Dichtung wie auch in der mediävistischen Forschung, vgl. Braun.

38 Zur, virtual polyphony‘, vgl. Deeming, S. 67.

39 Behr, S. 98.

40 Fassler, S. 5, beschreibt die mittelalterliche Liturgie treffend als ,a great wheel turning slowly, just behind the times“.

41 Die geistlichen Lieder, Anmerkungen, S. 206-208. 
lob sei ir von mir gesait,

gelobt sei all ir wirdikait. (V, 5-8)

Wo im restlichen Text die Aussagen aus einer unbestimmten Perspektive kamen, wird hier die Ich-Perspektive angenommen und die Figur des Komponisten nachdrücklich betont. Der Hinweis auf König David, seinen Gesang und sein Harfenspiel unterstreicht ebenfalls die Musikalität der Sequenz. Es spricht für sich selbst, dass diese Persönlichkeit, der biblische Sänger par excellence, gerade hier in den Vordergrund tritt, wo der Sequenztext die Ich-Perspektive explizit annimmt. Die implizite Identifizierung des Dichters erklärt vielleicht auch die sonst überraschende Bezeichnung dieses Liedes als psalmus in der Hs. F. Die Tendenz zur Personalisierung in Sälig sei der selden zeit hat einige Abschreiber anscheinend dermaßen irritiert, dass all mein frewde $(\mathrm{I}, 2)$ in den Hss. AGm durch all unser frewd ersetzt wurde. Die Ich-Aussagen im letzten Versikel blieben hingegen unangetastet, da wohl erkannt wurde, dass die Abweichung beabsichtigt war.

Wie notwendig es ist, Mundi renovatio unter Sälig sei der selden zait mitzuhören und sogar mitzufühlen, wird deutlich in der ausführlichen Rubrik der Hs. F.: Sequitur de beata virgine psalmus pasca sub melodia mundy renouacia [sic]. Dieser Überlieferungsträger weist nämlich keine musikalische Notation auf. Der Schreiber hat sich folglich nicht auf die Melodie stützen können, um dem Rezipienten die Totalität des Liedes vorzuführen. Wohl aus diesem Grunde werden die meisten der zwanzig geistlichen Lieder in der Handschrift mit lateinischen Rubriken versehen, die sie entweder als sub melodia oder secundum textum klassifizieren. ${ }^{42}$ Der zweite Fall fokussiert eher auf die genaue Wiedergabe des textlichen Inhalts, der erste Fall hingegen orientiert sich vor allem an der Melodie der Vorlage und rekurriert auf sie als sinnstiftenden Bezugspunkt. ${ }^{43}$ Die Kontrafaktur baut nämlich wesentlich auf dem Bedeutungspotenzial der Melodie auf, die aber - wie das Klangliche im Allgemeinen - der Nachwelt und der Forschung nicht immer einsichtig ist. Wieder handelt es sich um eine Überbrückung der Ellipse zwischen Text und Klangraum, die sich der Theorie entzieht.

Eine attraktive theoretische Antwort auf diese kritische Diskrepanz hat Susan Sontag 1964 im Schlusswort ihres Aufsatzes, Against Interpretation' angeboten. Sie diskutiert die Nutzbarkeit der althergebrachten Hermeneutik als Herangehensweise an die Kunst der Nachkriegszeit und schlägt vor, einen Neuansatz zu wagen, der von dem Konzept der Erotik ausgeht. Indem Sontag die Sinne und das

42 Achtzehn der Lieder stammen aus dem Mönch von Salzburg-Korpus. Zwei unter ihnen (G13: Mittit ad virginem und G28: Mundi renovacio) sind neben den Verdeutschungen Oswalds von Wolkenstein überliefert.

43 Zur Deutung der secundum textum und sub melodia-Etiketten, vgl. März, S. 296. 
Erlebnis des Rezipienten in den Vordergrund rückt, ist ihr Ansatz auch gut auf das geistliche Lied als Gesamtphänomen übertragbar.

What is important now is to recover our senses. We must learn to see more, to hear more, to feel more. [...] The aim of all commentary on art now should be to make works of art - and, by analogy, our own experience - more, rather than less real to us. The function of criticism should be to show how it is what it is, even that it is what it is, rather than to show what it means. In place of a hermeneutics we need an erotics of art. ${ }^{44}$

Dieses Plädoyer für eine Erotik der Kunst hat wohl zum Boom der Sound Studies in den letzten Jahren beigetragen. In gewisser Hinsicht reformuliert Sontags Appell aber nur einen seit der Antike existierenden ästhetischen Diskurs, dem die Termini dulcedo bzw. suavitas zugrundeliegen, für die Moderne. Die ,Süße‘ als höchstes ästhetisches Ziel bzw. preisendes Attribut ist ein roter Faden, der sich durch die Gedankenwelt des gesamten Mittelalters zieht. ${ }^{45}$ Beide Ansätze repräsentieren nämlich den Versuch, in der Kunstkritik einen Platz zu schaffen für Dinge, die zwar kaum zu erfassen sind, sich aber gleichwohl harmonisch in einen Gesamtzusammenhang einfügen. Die Tatsache, dass diese ,Süße“ nur schwer zu fassen ist, macht sie zum idealen Sinnbild des kaum analysierbaren Beitrags der Melodie zu einem Lied bzw. zum Gesang im Allgemeinen. Auch dies ist keine neue Idee. Die dulcedo wurde bereits seit dem neunten Jahrhundert mit der musikalischen Aufführung und besonders mit dem Singen verbunden. Aurelianus Reomensis hat etwa im fünften Kapitel seiner Musica disciplina den Anklang von mel in melodia hervorgehoben: Eufonia est suavitas vocis, haec et melos a suavitate vocis et melle dicta est, unde et melodia dicitur. ${ }^{46}$ Ebenfalls treffend ist eine Bemerkung Bernhards von Clairvaux, die die stimmliche Süße explizit auf das geistlichen Singen bezieht: Iesus mel in ore, in aure melos, in corde iubilus. ${ }^{47}$ Sowohl in der jüngeren wie auch der älteren Auffassung steht die sinnliche Kohärenz eines künstlerischen Produkts im Vordergrund. Es ist die Harmonie (im übertragenen Sinne), die den Erfolg und damit den Wert eines Liedes ausmacht. Dabei kann sie zugleich wesentlich zum religiösen Wert des jeweiligen Liedes beitragen.

Unter diesem Blickwinkel wende ich mich wieder dem Sälig sei der selden zeit zu. In diesem Fall mündet die Entscheidung, einen neuen Liedtext über die Gottesmutter zu komponieren und ihre Rolle in der Ostergeschichte mithilfe der Melodie einer bekannten Sequenz neu zu deuten, in eine neue Liedeinheit sowie in eine verschobene Vorstellung von Maria. Die Bausteine dieser neuen Verbin-

44 Sontag, S. 14 (Hervorhebung der Autorin).

45 Vgl. Carruthers.

46 Aurelianus Reomensis, Kap. 5-6, S. 69.

47 Bernhard von Clairvaux: 15.6, 1:86. 
dung waren aber ausdrücklich nicht mit einem intellektuellen, sondern mit einem affektiven Wert beladen. Margot Fassler hat dargelegt, wie entscheidend das Gewebe melodischer Wiederholungen und Variationen zwischen einzelnen Sequenzen für ihren Wert als System war. ${ }^{48}$ Wie Fassler anhand des viktorinischen Sequenzenrepertoires demonstriert, waren diese Beziehungen nicht nur musikalisch bedeutend, sondern hatten auch gemeinschaftsstiftende Kraft und spiegelten die Ordensgeschmeinschaft als Körper wider. ${ }^{49}$ Diese Beziehungen verdeutlichen eindrucksvoll die Rolle, die emotionale Assoziationen in der mittelalterlichen Liedkultur hatten. Eng mit Melodie und Klang verbunden, untermauerten sie die ,Süße' und damit den Wert eines Lieds.

\section{Kontrafakturen weltlicher Vorlagen}

Als letzten Schritt ziehe ich einen Fall heran, in dem es keine erkennbare Beziehung zwischen dem Salzburger Lied und dem Text seiner vermuteten melodischen Vorlage gibt. Diese weicht nicht nur im Inhalt ab, sondern gehört auch zur Gattung des weltlichen Lieds. Wie Gisela Kornrumpf aufgezeigt hat, ist Maria pis gegrüsset (G 12) - eine strophenweise Auslegung des Ave Maria - eine Kontrafaktur des einstrophigen Minnelieds Wernhers von Hohenberg Mich jamert $\hat{u} z$ der mâze (SMS XXVI, 4). ${ }^{50}$ Angesichts der eingangs besprochenen wissenschaftlichen Gewohnheit, Text und Musik strikt auseinanderzuhalten, könnte man dazu neigen, Maria pis gegrüsset als treffendes Beispiel für eine unüberbrückbare Kluft im Herzen der Liedkultur zu betrachten. Das muss aber nicht unbedingt der Fall sein. Die suavitas, die als Leitziel des mittelalterlichen Kunstwerkes im Allgemeinen und des Liedes im Besonderen aufgefasst werden kann, erlaubt nämlich großzügigen Raum für signifikante Unterschiede. Sie kann nicht nur als schön abgestimmte Süßigkeit verstanden, sondern auch als Salz beschrieben werden. ${ }^{51}$ Hier handelt es sich nicht um die willkürliche Heranziehung einer beliebigen ,schönen“ Melodie, sondern um ein Kunstwerk, das seine Wirkung aus dem dichten Netzwerk der Liedkunst bezieht.

Dieses Netzwerk schließt sowohl Weltliches wie auch Geistliches ein, und dieser Brückenschlag ist besonders prägnant im Fall des Salzburger Korpus, das,

48 Fassler, S. 300: ,[...] the entire interrelated complex resonated with a host of associations. A mind which contained all three sequences [sc. Dulce lignum, Laudes crucis und Zima vetus] would hear all three of them each time any one of them was sung“.

49 Fassler, S. 267-269.

50 Wachinger: Der Mönch von Salzburg. Zur Überlieferung, S. 127, Anm. 11.

51 Carruthers, S. $1000 \mathrm{f}$. 
wie eingangs bemerkt, am Hof des anscheinend nicht übermäßig frommen Erzbischofs Pilgrim II. von Puchheim enstand. ${ }^{52}$ Dies lässt sich gut anhand der Lieder Magd hochgeporn (G 20) und In gotes namen (G 38) darlegen. ${ }^{53}$ Das erste ist ein Mariengebet aus der Wir-Perspektive. Jede Strophe fokussiert eine bestimmte Szene aus der Heilsgeschichte, und alle Strophen schließen mit einer Anrufung Mariens. Das Stück ähnelt somit insgesamt der lateinischen Hymnendichtung. Das zweite Lied, ebenfalls aus der Wir-Perspektive verfasst, ist eine lehrhafte Auflistung der eucharistischen Mysterien und betont die Notwendigkeit, ein gutes und reines Leben zu führen, um sælde erlangen zu können. Gisela Kornrumpf hat darauf aufmerksam gemacht, dass beide Lieder dasselbe metrische Raster wie Gottfrieds von Neifen Seht an die heide (KLD 15, XX) aufweisen. ${ }^{54}$

$\begin{array}{llll}A & A & B & A \\ 2 a-3 b-3 a-3 b 3 a-3 a-3 c & 2 a-3 b-3 a-3 b 3 a-3 a-3 c & 2 d-3 e & 2 d-3 e-3 d-3 e 3 d-3 e-3 c\end{array}$

Die Kolmarer Liederhandschrift nennt dieses Raster dez muches hoffdon (München, BSB, Cgm 4997, fol. 645') und unterstützt damit die Erwartung einer Melodie aus dem weltlichen Bereich. Wie Stefan Rosmer jedoch kürzlich dargelegt hat, ist die melodische Form des Liedes (AABA) eher der lateinischen Liedkunst als den Meisterliedern oder dem Minnesang verwandt. ${ }^{55}$ Er demonstriert, wie die D-f Harmonik des Stegs mit der C-g Harmonik der Stollen kontrastiert und dass sich der Melodieanfang am achten Psalmton orientiert. Diese beiden Charakteristika wie auch die Einheitlichkeit der Melodie weisen auf eine ursprünglich klerikale Herkunft hin. Der wahrscheinliche Ausgangspunkt war also vermutlich nicht das bekannte volkssprachliche Lied. Entweder gab es eine andere Vorlage oder eine lateinische Zwischenstufe oder die Melodie ist speziell für das Magd hochgeporn und das In gotes namen komponiert worden. Im ersten Fall würde sich also der Versuch bemerkbar machen, die Resonanzen eines wohl lateinischen Liedes auf den neuen Liedtext des Mönchs zu übertragen. Im zweiten Fall (wenn die Melodie eine Komposition des Mönchs war) hieße das, dass der Mönch die Resonanzen einer ganzen Liedtradition auf ein bestehendes Raster übertragen wollte. In jedem Fall sind die Melodie und der Einsatz von Anklängen durch den Mönch integraler Bestandteil der kaleidoskopischen - oder besser kaleidophonischen - Darbietung

52 Zum erzbischöflichen Hof, vgl. Rosmer: Höfische Liedkunst im Kloster, und Schneider, S. $77-90$.

53 Editionen: Die geistlichen Lieder: G 20, S. 215-218; G 38, S. 296-300.

54 Kornrumpf: Bespr. zu Walther Röll, S. 19.

55 Ich möchte an dieser Stelle meine Dankbarkeit an Herrn Rosmer ausdrücken, der mir freundlicherweise einen Einblick in seine Monographie gegeben hat. 
dieser Lieder. Es ist dementsprechend Rosmers große Leistung, die Lieder aus dem Mönch von Salzburg-Korpus im Kontext einer breiten und bunten Liedkunst darzustellen, die weit über die weltlich-geistliche Grenze hinausgeht und in einer reinen Textanalyse verlorengegangen wäre. ${ }^{56}$

Dieser breite Horizont lässt sich ebenfalls am Beispiel des Maria pis gegrüsset veranschaulichen, das, wie bereits erwähnt, auf eine weltliche Formvorlage zurückgeht. Das Lied teilt nämlich die ,ungewöhnliche‘ Strophenform mit dem einstrophigen Minnelied Wernhers von Hohenberg Mich jâmert ûz der mâze, das aus dem frühen vierzehnten Jahrhundert stammt. ${ }^{57}$

Mich jâmert ûz der mâze

nâch der vil lieben vrouwen min.

got alle die verwâze,

dur die ich schiuhen muoz

ir wîplîch zartez bilde

ir mündel rât, ir wengel schîn:

sol mir daz wesen wilde,

dâ bî ir friuntlich gruoz,

ir kinne, ir kel, ir goltvar hâr,

ir hende, ir arme blanc,

ir lîp, ir nas, ir ougen klâr,

sol ich daz lange mîden,

sô muoz ich kumber lîden

und wirde an fröiden kranc. (SMS XXVI, 4)
1

5

10

Die Beziehung zwischen Maria und dem wîp mit dem zarten bilde ist nur oberflächlich: Die Charakteristika der lieben vrouwen werden etwas unsystematisch vorgeführt, und das Lied könnte vielleicht als Bitte um weibliche Intervention beschrieben werden. Die suavitas und der Genuss dieser Lieder fußen aber vor allem auf den Sinnen und erst danach auf dem Intellekt. ${ }^{58}$ Hören wir also zuerst der Melodie zu. Ich gebe Text und Melodie nach der Handschrift D wieder, auf der Spechtler seine Ausgabe aufbaut.

56 Haug, S. 99, vermutet ähnliche Übereinstimmungen zwischen den geistlichen und jetzt verlorenen weltlichen Liedern Peter Abaelards.

57 Schiendorfer, Sp. 938.

58 Carruthers, S. 999, betont die mittelalterliche Auffassung, derzufolge die Ästhetik zwischen der sinnlichen und der intellektuellen Ebene vermittelte. 

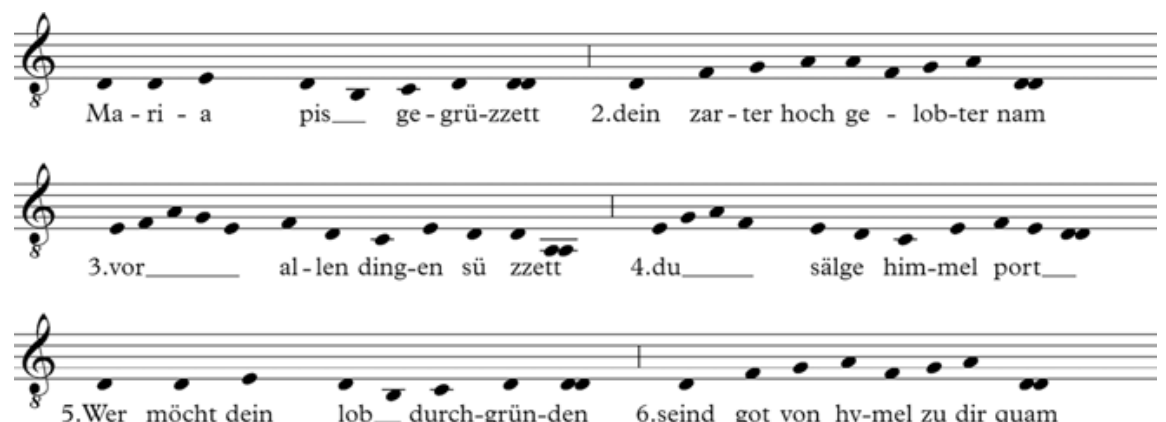

5.Wer möcht dein lob_durch-grün-den 6.seind got von hy-mel zu dir quam
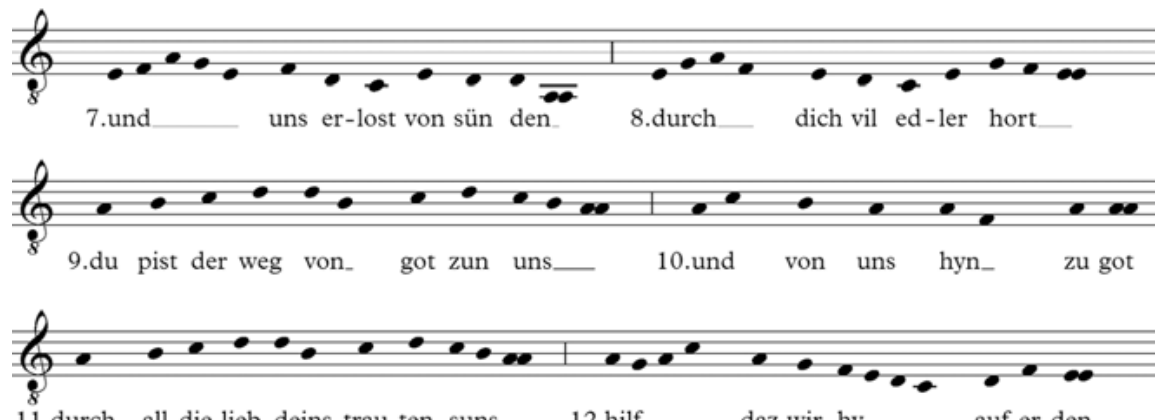

11.durch all die lieb deins trau-ten suns_ 12.hilf___ daz wir hy___ auf er-den

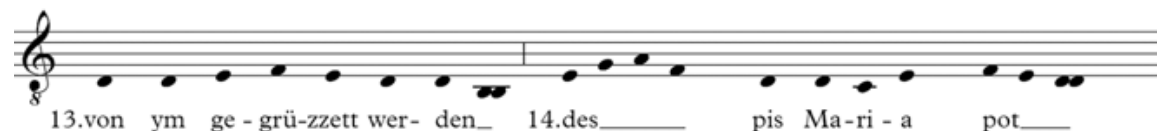

Notenbeispiel 2: Maria pis gegrüsset (G12) in Text und Musik nach Hs. D

II Genaden hast du funden,

die Eva uns verloren hat,

gib wider, frau, zu stunden,

wann unser ist dein fund.

durch uns pist gereichet

das got durch dich tut und auch lat, ,

das nieman dir geleichet,

das ist an dir wol kund.

du hast genad auch und gewalt

mit uns zu aller frist,

dein zärtleich junkfräulich gestalt

sol uns genad erwerben,

erwend uns ewigs sterben

seind du genädig pist. 
III Got ist mit dir verainet,

das er dem sünder zürnet nit, den dein genad wol mainet, darumb ward er dein kint. wie oft wir sünd begingen, das wir denn durch dein fleglich pit genad von im enpfingen, die niemand an dich fint.

o wie gar selig küssen drukt dein mund an kindleins mund, do er sich an dein brüstlein smukt und saugt an deinem herzen. man in an kindlich scherzen, sprich: ,pis mit in all stund'.

\section{Du pist ob allen weiben}

gesegnet, das die warhait muss

das wunder von dir schreiben, das muter magt mag sein und wort zu fleisch ist worden, da prach an dir der englisch gruss den natürlichen orden,

das nie an weib ward schein.

dein vater ist dein kind mit recht, das wold got sein durch dich, der edlist herr ist worden knecht, der für uns hat geliten, durch den, frau, wir dich piten: gesegn uns ewiklich.

V Deins leibes frucht geseget

ist, Jesus Christ, der cih verparch

zu dir vil rainer meget,

der uns durch dich erlost.

du pist frau aller engel, der trinitat ain edler sarch, der selikait umbvengel, des sünder höchster trost.

dich pitt die ellen christenhait

in disem jamertal, gib uns zu deiner frucht gelait, das wir in gotes namen zu himel varen, amen, in aller heiligen zal.

Die Melodie von Mich jamert ûz der mâze ist in vielerlei Hinsicht ungewöhnlich. Sie hat erstens eine außergewöhnlich breite Spanne von $A$ bis $d^{\star}$, die auch extrem 
tief beginnt: Töne unter $c$ treten im sonstigen Mönchs-Korpus sehr selten auf. Auffällig ist zweitens ihre Konstruktion aus leicht variierenden und mehrmals wiederkehrenden Einheiten. So tritt beispielsweise eine Variation der Melodie des Verses 3 ( $y$ im Schema unten) fünfmal hervor. In den Versen 4 und 8 endet die Notenfolge jeweils um eine Quarte und Quinte höher und erscheint letztlich in vereinfachter Form im letzten Vers der Strophe. Die Wiederholung der Melodie des zweiten bzw. vierten Verses ( $\beta$ im Schema unten) um eine Sexte höher im Abgesang trägt ebenfalls zur Einheitlichkeit der Melodie bei. Die Explikation eines Verses des Ave Maria, die in jeder Strophe erfolgt, führt also musikalisch nicht weit vom kanonischen Ausgangspunkt weg. Sie entspricht auf diese Weise dem wiederkehrenden c-Reim, der die Stollen zusammenbindet. Die einheitliche Struktur der Melodie mit ihrer progressiven Wiederholung eignet sie besonders gut für die piae exercitiae.

\begin{tabular}{|c|c|c|c|c|c|c|c|c|c|c|c|c|c|c|}
\hline \multirow[b]{2}{*}{ Vers } & \multicolumn{4}{|c|}{ Stollen 1} & \multicolumn{4}{|c|}{ Stollen 2} & \multicolumn{6}{|c|}{ Abgesang } \\
\hline & 1 & 2 & 3 & 4 & 5 & 6 & 7 & 8 & 9 & 10 & 11 & 12 & 13 & 14 \\
\hline G 12 & $4 a$ & $4 b$ & $4 a$ & $4 \mathrm{C}$ & $4 d$ & $4 \mathrm{e}$ & $4 d$ & $4 C$ & $4 f$ & $3 g$ & $4 f$ & $3 h^{6}$ & $3 h^{6}$ & $4 \mathrm{~g}$ \\
\hline Wernher & $4 a^{\prime}$ & $4 b$ & $4 a^{6}$ & $4 c$ & $4 d^{\circ}$ & $4 b$ & $4 d^{\prime}$ & $4 C$ & $4 e$ & $3 \mathrm{f}$ & $4 e$ & $3 g^{6}$ & $3 \mathrm{~g}^{6}$ & $4 \mathrm{f}$ \\
\hline Melodie & $\alpha$ & $\beta$ & Y & $y^{\prime}$ & $\alpha$ & $\beta$ & $\gamma$ & $\gamma^{\prime}$ & $\beta^{\prime}$ & $\delta$ & $\beta^{\prime}$ & $\varepsilon$ & $\zeta$ & $y^{\prime}$ \\
\hline
\end{tabular}

Die suavitas der neuen Liedeinheit im Korpus des Mönchs von Salzburg liegt also in der Zusammensetzung von Elementen, die die Charakteristika des Vorlagelieds neu werten und erfolgreich ausnutzen kann. Dies geschah nicht nur im Salzburger Fall, sondern mehrfach in der langen Geschichte dieses Rasters und der verwandten Melodie. Das metrische Raster von Mich jâmert ûz der mâze bzw. Maria pis gegrüsset kommt in zwei anderen Fällen ebenfalls vor: in drei Sprüchen im St. Marienthaler Psalter, die wohl von Wernher stammen, und in zwei Mariengebeten aus dem Thurgauer Kloster St. Katharinenthal, die in der ersten Hälfte des 14. Jahrhunderts entstanden. ${ }^{59}$ Dieser Beispielfall steht damit metonymisch für die komplexe Sinnstiftung in der mittelalterlichen Liedkunst, deren Motor überwiegend musikalisch war.

59 Zur Datierung des St. Marienthaler Psalters, Kornrumpf: Drei unbekannte Sangsprüche, S. 87. Die Mariengebete sind ediert worden in Kornrumpf: Vom Codex Manesse, Bd. 2, B. 8. I-II. 


\section{Fazit. Ein ,volles Lied“}

Nach den vorausgehenden Analysen ist es wenig überraschend, dass der Mönch in der dritten Strophe von In gotes namen (G 38) das Gehör als herausragenden und glaubwürdigsten Sinn preist. ${ }^{60}$

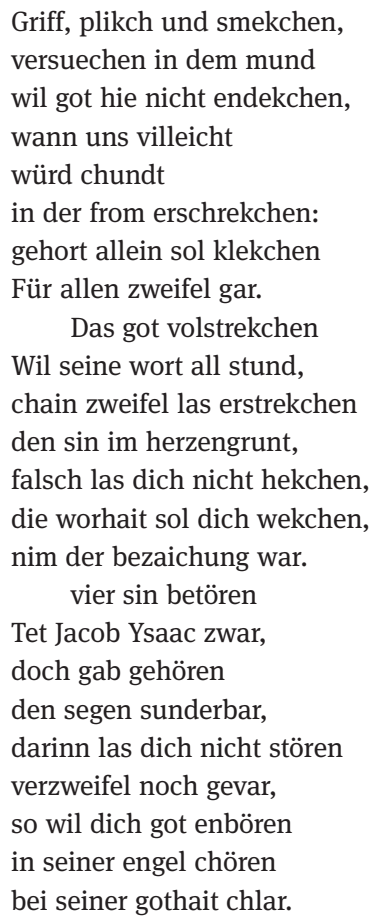

Ich habe versucht zu veranschaulichen, wie das Gehör auch in Analyse und Interpretation der leitende Sinn sein sollte. Lauscht man nämlich aufmerksamer, kann man das hören, was ich als ein ,volles Lied“ bezeichnen möchte. ${ }^{61}$ Ich habe diesen Beitrag mit einem Übertragungsverfahren eröffnet, das den Abstand zwischen Sprache, Melodie und Klang genau dort überbrückt, wo der körpernahe Wert der erklingenden Handlung sitzt. Das ,volle Lied‘ beschränkt sich nicht nur auf sein hermeneutisches Potenzial, sondern schließt im Sinne von Susan Sontag auch den ,erotischen' Wert seiner Form und seiner geistigen und klingenden Kontur mit ein. In meinem zweiten Beispiel - Mundi renovatio - wurden Sprache

60 Die geistlichen Lieder, S. $297 \mathrm{f}$.

61 Vgl. Stock. 
und Melodie voneinander abgekoppelt, damit der Dichter einen neuen Konnex zwischen Maria und Ostern erstellen konnte. Dieser Konnex verlässt sich auf das Rüstzeug der Melodie für seine neue komplexe Bedeutung.

Das ,volle Lied' bedeutet also, dass man Text und Melodie nicht als separate Eigenheiten auffasst, sondern seine lebensweltlich verwurzelte Existenz als Teil einer oder mehrerer klanglicher Traditionen miteinbezieht. Das ,volle Lied“ schließt (wie Zumthors œuvre) ebenfalls seine Verwirklichung in der Aufführung ein und berücksichtigt auch das Netz der Resonanzen und Anklänge, die im Rezipienten ausgelöst werden. Das habe ich mit meinem dritten Beispiel vorgeführt. Wer Lieder im eigentlichen Sinne als Lieder behandeln will, muss ein breites Spektrum an Elementen einbeziehen, die aufgrund ihrer Flüchtigkeit und subjektiven Natur oft nur schwer greifbar sind. Der Versuch lohnt sich aber, wenn dadurch das Lied in seiner Fülle zum Erklingen gebracht werden kann.

\section{Literaturverzeichnis}

Arlt, Wolf: Das eine Lied und die vielen Lieder. Zur historischen Stellung der neuen Liedkunst des frühen 12. Jahrhunderts. In: Fs. Rudolf Bockholdt zum 60. Geburtstag. Hg. von Norbert Dubowy, Sören Meyer-Eller. Pfaffenhofen 1990, S. 113-129.

Aurelianus Reomensis: Musica disciplina. Hg. von Lawrence Gushee. [o.0.], 1975 (Corpus scriptorum de musica 21).

Bärnthaler, Günther: Die Hymnen- und Sequenzenübersetzungen des Mönchs von Salzburg. Diss. Masch. Salzburg 1977.

Behr, Hans-Joachim: Stabat mater dolorosa. Zum Verhältnis von Vorlage und Bearbeitung in der Lieddichtung des Mönchs von Salzburg. In: ZfdA 116 (1987), S 83-99.

Bernhard von Clairvaux: Sermones super Cantica canticorum. In: S. Bernardi opera. Hg. von Jean Leclercq u. a. Bd. 1. Rom 1957.

Braun, Manuel: Aufmerksamkeitsverschiebung. Zum Minnesang des 13. Jahrhunderts als Formund Klangkunst. In: Wolfram-Studien XXI (2013), S. 203-230.

Carruthers, Mary: Sweetness. In: Speculum 81 (2006), S. 999-1013.

Deeming, Helen: Music, Memory and Mobility. Citation and Contrafactum in Thirteenth-Century Sequence Repertoires. In: Citation, Intertextuality and Memory in the Middle Ages and Renaissance. Bd. 2: Cross-Disciplinary Perspectives on Medieval Culture. Hg. von Giuliano di Bacco, Yolanda Plumley. Liverpool 2013, S. 67-81.

Der Mönch von Salzburg. Die Melodien zu sämtlichen geistlichen und weltlichen Liedern. Hg. von Hans Waechter, Franz Viktor Spechtler. Göppingen 2004 (GAG 719). Die geistlichen Lieder des Mönchs von Salzburg. Hg. von Franz Viktor Spechtler. Berlin, New York 1972 (Quellen und Forschungen zur Sprach- und Kulturgeschichte der germanischen Völker NF 51).

Die Schweizer Minnesänger. Hg. von Karl Bartsch. Frauenfeld 1886. Ndr. Darmstadt 1964.

Die weltlichen Lieder des Mönchs von Salzburg. Texte und Melodien. Hg. von Christoph März. München 1999 (MTU 114).

Dillon, Emma: The Sense of Sound. Musical Meaning in France, 1260-1330. Oxford 2012. 
Emmelius, Caroline: ,süeze stimme, süezer sang‘. Funktionen von stimmlichem Klang in Viten und Offenbarungen des 13. und 14. Jahrhunderts. In: Zeitschrift für Literaturwisssenschaft und Linguistik 43. Jg., 171 (2013), S. 64-85.

Engels, Stefan: Geistliche Lieder des Mönchs von Salzburg. In: Musikleben des Spätmittelalters in der Region Österreich. Hg. von Birgit Lodes u. a. https://musical-life. net/essays/geistliche-lieder-des-moenchs-von-salzburg (21. April 2019).

Fassler, Margot E.: Gothic Song. Victorine Sequences and Augustinian Reform in Twelfth-Century Paris. 2. Aufl. Notre Dame 2011.

Finscher, Ludwig: Einleitung. In: Die Musik des 15. und 16. Jahrhunderts. Bd. I. Hg. von Ludwig Finscher. Laaber 1989.

Gibbs, Raymond: Embodied Experience and Linguistic Meaning. In: Brain and Language 84 (2003), S. 1-15.

Gruber, Jörn: Die Dialektik des Trobar. Untersuchungen zur Struktur und Entwicklung des okzitanischen und französischen Minnesangs des 12. Jahrhunderts. Tübingen 1983 (Beihefte zur Zeitschrift für romanische Philologie 194).

Harper, Sally, P.S. Barnwell, Magnus Williamson (Hgg.): Late Medieval Liturgies Enacted. The Experience of Worship in Cathedral and Parish Church. London 2017.

Haug, Andreas: Das Mittelalter. In: Musikalische Lyrik. Teil I: Von der Antike bis zum Mittelalter. Hg. von Herman Danuser. Laaber 2004, S. 52-129.

Hirschberg, Dagmar: ,dy trumpet‘ (MR 15). Ein Tagelied-Experiment des Mönchs von Salzburg. In: Lied im deutschen Mittelalter. Überlieferung, Typen, Gebrauch. Chiemsee-Colloquium 1991. Hg. von Cyril Edwards u. a. Tübingen 1996, S. 203-213.

Janota, Johannes: Studien zu Funktion und Typus des deutschen geistlichen Liedes im Mittelalter. München 1968 (MTU 23).

Jones, Claire Taylor: Christian Listening and the Ethical Community of the Liturgical Text. In: Literature and Theology 27 (2013), S. 227-239.

Kandler, Johannes: ,Gedoene ân wort daz ist ein tôter galm‘. Studien zur Wechselwirkung von Wort und Ton in einstimmigen Gesängen des hohen und späten Mittelalters. Wiesbaden 2005 (Elementa musicae 5).

Köbele, Susanne: Rhetorik und Erotik. Minnesang als ,süßer Klang‘. In: Poetica 45 (2013), S. $299-332$.

Kornrumpf, Gisela, Burghart Wachinger: Alment. Formentlehnung und Tönegebrauch in der mittelhochdeutschen Spruchdichtung. In: Gisela Kornrumpf: Vom Codex Manesse zur Kolmarer Liederhandschrift. Aspekte der Überlieferung, Formtraditionen, Texte. Tübingen 2008 (MTU 133), S. 117-168.

Kornrumpf, Gisela: Bespr. zu Walther Röll: Vom Hof zur Singschule. Überlieferung und Rezeption eines Tones im 14.-17. Jahrhundert. In: Anzeiger für deutsches Altertum 90 (1979), S. 14-22.

Kornrumpf, Gisela: Drei unbekannte Sangsprüche des 13. Jahrhunderts. In: Der St. Marienthaler Psalter. Hg. von Helmut Engelhart. Regensburg 2006, S. 79-87.

Kornrumpf, Gisela: Vom Codex Manesse zur Kolmarer Liederhandschrift. Aspekte der Überlieferung, Formtraditionen, Texte. 2 Bde. München 2008 (MTU 133).

Kraß, Andreas: ,Mittit ad virginem‘. Bearbeitungen der Mariensequenz durch den Mönch von Salzburg, Oswald von Wolkenstein und Heinrich Laufenberg. In: Maria in Hymnus und Sequenz. Interdisziplinäre mediävistische Perspektiven. Hg. von Eva Rothenberger, Lydia Wegener. Berlin, Boston 2017 (Liturgie und Volkssprache 1), S. 193-219. 
von Kraus, Carl: Deutsche Liederdichter des dreizehnten Jahrhunderts. 2. Aufl. Hg. von Gisela Kornrumpf. Tübingen 1978.

März, Christoph: Pange lingua per omnia verba et melodia. Zu den Anfängen poetischer Hymnennachdichtung in deutscher Sprache. In: Der lateinische Hymnus im Mittelalter. Überlieferung - Ästhetik - Ausstrahlung. Hg. von Andreas Haug u. a. Kassel u. a. 2004 (Monumeta Monodica Medii Aevi. Subsidia 4), S. 279-299.

Murray, David: The Clerical Reception of Bernart de Ventadorn's ,Can vei la lauzeta mover' (PC 70, 34). In: Medium Ævvum 85 (2016), S. 259-277.

Quast, Bruno: Vom Kult zur Kunst. Öffnungen des rituellen Textes im Mittelalter und früher Neuzeit. Tübingen 2005 (Bibliotheca Germanica 48).

Reiffenstein, Ingo: Deutsch und Latein im Spätmittelalter. Zur Übersetzungstheorie des 14. und 15. Jahrhunderts. In: Fs. Siegfried Grosse zum 60. Geburtstag. Hg. von Werner Besch u.a. Göppingen 1984 (GAG 423), S. 195-208.

Rosmer, Stefan: Der Mönch von Salzburg und das lateinische Lied. Die geistlichen Lieder in stolligen Strophen und das einstimmige gottesdienstliche Lied im Spätmittelalter. Wiesbaden 2019 (Imagines Medii Aevi 44).

Rosmer, Stefan: Höfische Liedkunst im Kloster, in der Stadt und andernorts. Zur Rezeption der geistlichen Lieder des Mönchs von Salzburg in der ersten Hälfte des 15. Jahrhunderts. In: Musikalische Repertoires in Zentraleuropa (1420-1450). Prozesse und Praktiken. Hg. von Alexander Rausch, Björn R. Tammen. Wien 2014, S. 271-297.

Schadendorf, Marjam: Zum Verhältnis von Text und Melodie in den Liedern KL 33-36. In: Jahrbuch der Oswald von Wolkenstein Gesellschaft 9 (1996/1997), S. 239-257.

Schiendorfer, Max: Art. ,Werner von Hohenberg‘. In: VL² 10 (1998), Sp. 936-940.

Schneider, Almut: Er liez ze himel tougen erhellen sîner stimme dôn. Sprachklang als poetische Fundierung normativen Sprechens. In: Text und Normativität im deutschen Mittelalter. XX. Anglo-German Colloquium. Hg. von Elke Brüggen u.a. Berlin 2012, S. $199-216$.

Schneider, Almut: Poetik des Klangs. Dichtkunst im Diskurs der ,musica‘ in Texten des deutschen Mittelalters. Habilitationsschrift Eichstätt 2012.

Sontag, Susan: Against Interpretation. In: dies.: Against Interpretation. Ndr. London 1994, S. 3-14.

Stock, Markus: Das volle Wort. Sprachklang im späteren Minnesang. Gottfried von Neifen, ,Wir suln aber schône enpfâhen“ (KLD Lied 3). In: Text und Handeln. Zum kommunikativen Ort von Minnesang und antiker Lyrik. Hg. von Albrecht Hausman. Heidelberg 2004 (Beihefte zum Euphorion 46), S. 185-202.

Straub, Henriette: ,Mundi renovatio“ und ,Mittit ad Virginem‘ translated by the ,Monk of Salzburg' and Oswald von Wolkenstein. In: Jahrbuch der Oswald von Wolkenstein Gesellschaft 9 (1996/97), S. 509-522.

Szövérffy, Joseph: Marianische Motivik der Hymnen. Ein Beitrag zur Geschichte der marianischen Lyrik im Mittelalter. Leiden 1985 (Medieval Classics 18).

Wachinger, Burghart: Der Mönch von Salzburg. In: VL² 6 (1987), Sp. 658-670.

Wachinger, Burghart: Der Mönch von Salzburg. Zur Überlieferung geistlicher Lieder im späten Mittelalter. Tübingen 1989 (Hermaea NF 57).

Waechter, Hans: Die geistlichen Lieder des Mönchs von Salzburg. Untersuchungen unter besonderer Berücksichtigung der Melodien. Göppingen 2005 (GAG 724). 
Welker, Lorenz: Das Taghorn des Mönchs von Salzburg. Zur frühen Mehrstimmigkeit im deutschen Lied. In: Schweizer Jahrbuch für Musikwissenschaft 4/5 (1984/1985), S. 41-61.

Williamson, Beth: Sensory Experience in Medieval Devotion. Sound and Vision, Invisibility and Silence. In: Speculum 88 (2013), S. 1- 43.

Zumthor, Paul: Essai de poétique médiévale. Avec un préface de Michel Zink et un texte inédit de Paul Zumthor. Paris 2000. 
